\title{
Electrospun Cellulose Acetate Fiber Mats as Carriers for Crude Extracts From Phyllanthus Emblica Linn. Fruits
}

\author{
Patcharaporn Thitiwongsawet ${ }^{1, a}$, Thipawan Boonruang ${ }^{1}$, Thanchanok Noochsuparb ${ }^{1}$ \\ ${ }^{1}$ Department of Chemical Engineering, Faculty of Engineering, Thammasat University, Klong-Luang, Pathumthani. 12120. Thailand
}

\begin{abstract}
Fruit of Phyllanthus emblica Linn (PE) was extracted with methanol and then partitioned into diethyl ether, ethyl acetate, butanol, and water fractions. The diethyl ether fraction showed the highest antibacterial activity against two pathogenic bacteria including Escherichia coli (ATCC 25922) and Staphylococcus aureus (ATCC 25923). The PE extract from diethyl ether fraction was then loaded into $17 \% \mathrm{w} / \mathrm{v}$ cellulose acetate (CA) solution prepared in 2:1 $\mathrm{v} / \mathrm{v}$ acetone $/ N, N$-dimethylacetamide at concentrations of 1,3 , and $5 \% \mathrm{w} / \mathrm{w}$ (based on the weight of CA). The PE extract-loaded electrospun CA fiber mats were fabricated by electrospinning. The morphological appearance of both the neat and the PE extract-loaded electrospun CA fibers were smooth with the average diameters in a range of 335$694 \mathrm{~nm}$. The amounts of water retention and release characteristics of PE extract from fiber mats in an acetate buffer (pH 5.5) were studied and compared with the corresponding cast-films fabricated by the solvent casting method. The amount of water retention of the PE extract-loaded fiber mats was about $112-205 \%$ which was higher than that of the cast films (i.e. 25-35\%). The study of release characteristics of PE extract from fiber mats and films was carried out by total immersion method in an acetate buffer at $32^{\circ} \mathrm{C}$ for $72 \mathrm{~h}$. The PE extract-loaded fiber mats exhibited greater amount of extract released than those of the cast films. The maximum amounts of PE extract released from fiber mats and films were about $40-80 \%$ and $18-23 \%$, respectively.
\end{abstract}

\section{Introduction}

Phyllanthus emblica Linn (PE), also known as Indian gooseberry, emblic or amla, is in Euphorbiaceae family. The tree is widely distributed in deciduous forest in subtropical and tropical areas of Southeast Asia. PE has been used in traditional medicine since an ancient time for anti-inflammatory and antipyretic treatment. The chemical compositions of PE extracts are mainly phenolic compounds. Wei Luo et al. reported the chemical compositions of PE fruits methanolic extracts including gallic acid, ellagic acid, mucic acid 1,4-lactone 3-O-gallate, isocorilagin, chebulanin, chebulagic acid and mallotusinin [1]. The extracts of PE fruits [1-7] and leaves [7-9] has been reported to posses several pharmaceutical properties. These studies revealed that PE extracts exhibit antioxidation [4], antibacterial for both gram-positive and gram-negative [2-3, 5, 7-8], antiinflammatory and anti-pyretic [9], antifungal [2], antidiarrheal [6], and antiproliferative of cancer cell [2] properties.

Electrospinning is an interesting process for producing ultra-fine fibers with diameters in range of nanometers to submicrometers. Electrospun fibers have attracted a great deal of attention in many applications such as filtration of particles [10], composite reinforcement [11], scaffolds for tissue engineering [12], and drug delivery system [13-14], because of their high surface area-to-volume ratio and high porosity. One of the advantages of the electrospun fibers over the cast films as carriers for drugs is the highly porous nature of fibers which exhibit much greater surface area, assumingly allowing drug molecules to diffuse out from the matrix much more conveniently.

Cellulose acetate (CA), one of the common biopolymer, was chosen to use as carrier for the extract delivery in this study. Successful electrospinning of 12.5$20 \% \mathrm{w} / \mathrm{w}$ CA solutions into ultra-fine fibers was reported by Liu and Hsieh [15]. The suitable solvent system for preparing electrospun CA fibers were $2: 1 \mathrm{v} / \mathrm{v}$ acetone: $N, N$-dimethylacetamide, in which the size of resulting CA fibers were in range of $100 \mathrm{~nm}$ to $1 \mu \mathrm{m}$ [15]. Electrospun CA fibers are widely used as carrier of drugs because of their good tissue compatibility and ease of fabrication. Various types of vitamins [13] and antiinflammatory drugs [14] were incorporated into electrospun CA fiber mats. The potential of these drugloaded fiber mats for transdermal delivery have been reported. The incorporation of 2,6-dichloro-4-nitroaniline, a fungicide for postharvest control of rhizopus, into electrospun CA fiber mats were carried out and their release characteristics were reported [16].

In the present work, PE fruits were extracted and tested for antibacterial activities against Escherichia coli and Staphylococcus aureus. The PE extract was loaded into $\mathrm{CA}$ solution which was then prepared into ultra-fine

\footnotetext{
a Corresponding author: tpatchar@engr.tu.ac.th
} 
fibers by electrospinning. Morphology and water retention properties of the neat and PE extract-loaded fiber mats were investigated. The release characteristics of PE extract from the fiber mats were studied and compared with the corresponding cast films. Finally, the antibacterial activities of the PE extract-loaded fiber mats were studied.

\section{Experimental}

\subsection{Materials}

Indian gooseberry fruits were purchased from a local market in Pathumthani province. Cellulose acetate (CA; $\mathrm{M}_{\mathrm{w}} \approx 30,000 \mathrm{Da}$; acetyl content $=39.7 \mathrm{wt} \%$; degree of acetyl substitution $\approx 2.4$ ) was purchased from SigmaAldrich (Switzerland). Acetone, N,N-dimethylacetamide, sodium acetate, glacial acetic acid, methanol, and ethyl acetate were purchased from Carlo Erba, Italy. Butanol and diethyl ether were purchased from Ajax Finechem, Australia. All of chemical were of analytical reagent grade.

\subsection{Methods}

\subsubsection{Extraction of PE fruits.}

PE fruits were extracted by following the procedure reported by Xiaoli Liu et al. [2]. Fresh PE fruits were cleaned and seeds were removed. The fruits were dried in an oven at $40^{\circ} \mathrm{C}$ for $3 \mathrm{~h}$ and then were grounded. The obtained powder were shaken in $60 \% \mathrm{v} / \mathrm{v}$ methanol at a powder-to-methanol ratio of $1 \mathrm{~g}: 10 \mathrm{ml}$ at $50^{\circ} \mathrm{C}$ for $3 \mathrm{~h}$. Filtering of the extracts were performed by using muslin cloth and $0.45 \mu \mathrm{m}$ filter paper. The residue was reextracted by repeating the previous procedure. The filtrates were combined. The solvent was removed by using a rotary evaporator. The $20.0 \mathrm{~g}$ of methanolic extract was dissolved in $40 \mathrm{ml}$ distilled water and then partitioned by each $400 \mathrm{ml}$ of diethyl ether, ethyl acetate, and butanol, respectively. For each fraction, solvent was removed by using rotary evaporator. The yield of each fraction was calculated.

\subsubsection{Antibacterial activities of the PE extracts}

The antibacterial activity of each fraction was studied against two pathogenic bacteria including Escherichia coli (ATCC 25922) and Staphylococcus aureus (ATCC $25923)$ by agar disc diffusion method. The plates were incubated at $37^{\circ} \mathrm{C}$ for $24 \mathrm{~h}$. The clear zone was the observed and recorded. Tetracycline was used as a positive control.

\subsubsection{Preparation of the PE extract-loaded CA fiber mats and cast films}

A $17 \% \mathrm{w} / \mathrm{v}$ CA solution was prepared by dissolving a weight amount of CA powder in a mixed solvent of $2: 1$ v/v acetone $/ N, N$-dimethylacetamide. Only the diethyl ether fraction of PE extract was loaded into CA solution to prepare fiber mats and cast films. PE extracts were loaded into $\mathrm{CA}$ solutions at concentrations of 1,3 , and $5 \% \mathrm{w} / \mathrm{w}$ (based on the weight of the CA powder). The PE extract-loaded fiber mats were fabricated by means of electrospinning. A stainless steel needle with the outside diameter of $0.91 \mathrm{~mm}$ was used as a nozzle. A rotating collector was used to collect fiber mat at a rotational speed of about $60 \mathrm{rpm}$. A high voltage power supply (ES30P-5W) was used to generate a high DC potential. The electric field was $15 \mathrm{kV} / 15 \mathrm{~cm}$. The polarity of the emitting electrode was positive. The fibers were collected for $10 \mathrm{hr}$. The feed rate was controlled to about $1 \mathrm{ml} / \mathrm{h}$, by means of a syringe pump. The shear viscosity of each spinning solutions was determined using a Brookfield DV-II programmable viscometer at $25^{\circ} \mathrm{C}$. The PE extractloaded films were also prepared for comparative studies by solvent-casting technique at the same PE extract concentrations i.e., 1,3 , and $5 \% \mathrm{w} / \mathrm{w}$. The thickness of both fiber mats and films were in the range of 50-60 $\mu \mathrm{m}$.

\subsubsection{Characterization of the PE extract-loaded CA fiber mats and cast films}

The morphology of the neat and PE extract-loaded fiber mats was investigated by using a HITACHI S-3400N scanning electron microscope (SEM). The diameters of fiber segment were measured from the SEM images, using the SemAphore 4.0 software. The average diameter of fibers was calculated from at least 50 measurements. The amount of water retention of both fiber mats and cast films were determined after submersion in an acetate buffer ( $\mathrm{pH}$ 5.5) solution for $72 \mathrm{~h}$ according to the following equation:

Water retention $(\%)=\frac{M-M_{i}}{M_{i}} \times 100$

where $M$ is the weight of each sample after submersion in an acetate buffer solution for $72 \mathrm{~h}$. and $M_{i}$ is the initial weight of the sample in its dry state.

\subsubsection{Release of PE extract-loaded CA fiber mats and cast films}

Actual PE extracts content. The actual amounts of PE extract in the fiber mats and cast films were determined. Each specimen was cut in a square shape of $2 \mathrm{~cm} \times 2 \mathrm{~cm}$ and was dissolved in $5.0 \mathrm{ml}$ of $2: 1 \mathrm{v} / \mathrm{v}$ acetone $/ N, N$ dimethylacetamide. Then, $2.0 \mathrm{ml}$ solution was added into $4.0 \mathrm{ml}$ of the same solvent. The actual amounts of PE extract were determined by a HITACHI U-2900 UV-vis spectrophotometer at the wavelength of $287 \mathrm{~nm}(\lambda \max )$. The actual amounts of PE extract in fiber mats and films were calculated from the obtained data against a predetermined calibration curve for PE extract.

Release of PE extracts. The release characteristics of PE extract from the PE extract-loaded CA fiber mat and film specimens were investigated. A total immersion method was carried out. Each specimen (square shape of $2 \mathrm{~cm} \times 2 \mathrm{~cm}$ ) was immersed in $40 \mathrm{ml}$ of acetate buffer 
(pH 5.5) solution at $32^{\circ} \mathrm{C}$. At a specified immersion time point ranging between $5 \mathrm{~min}-72 \mathrm{~h}, 1.0 \mathrm{ml}$ of the solution was withdrawn and the same volume of the fresh buffer was added. The amounts of PE extract released from the specimens were determined by the UV-vis spectrophotometer at the wavelength of $287 \mathrm{~nm}$. The experiments were carried out in triplicate.

\section{Results and Discussions}

\subsection{Extraction of PE fruits and anitibacterial activities of the PE extracts}

Starting from $500 \mathrm{~g}$ of dried PE fruit powders, approximately a $100 \mathrm{~g}$ of methanolic crude extract was obtained. After partitioning with solvents, the percent yields of diethyl ether, ethyl acetate, butanol, and water fractions were about 2.4, 25.5, 34.6, and 33.7\%, respectively. Both methanolic crude extract and four fractions were then studied for antibacterial activity against $E$. coli and $S$. aureus by agar disc diffusion method. The antibacterial activity was determined by the mean diameter of inhibition zone as shown in Table 1. The diethyl ether fraction showed the highest antibacterial activity against $S$. aureus, followed by ethyl acetate, butanol, water fractions, and methanolic crude extract, respectively. Quite similar trend was observed for the antibacterial activity against $E$. coli. The diethyl ether fraction also exhibited the largest inhibition zone, followed by ethyl acetate, and butanol fractions, respectively. However, the water fraction and methanolic crude extract did not show inhibition zone. It is obvious that diethyl ether fraction exhibited the greatest antibacterial activities against both $S$. aureus and E. coli; therefore this fraction was chosen to load into CA solution and fabricate into fiber mats in the next section.

Table 1. Antibacterial activities of the PE extracts against $E$. coli and $S$. aureus

\begin{tabular}{|c|c|c|}
\hline \multirow{2}{*}{ Sample } & \multicolumn{2}{|c|}{$\begin{array}{c}\text { Mean diameter of } \\
\text { inhibition zone [mm] }\end{array}$} \\
\cline { 2 - 3 } & E. coli & S. aureus \\
\hline Methanolic crude extract & 0 & $14.0 \pm 0.4$ \\
\hline Diethyl ether fraction & $4.4 \pm 0.1$ & $19.8 \pm 0.5$ \\
\hline Ethyl acetate fraction & $3.0 \pm 0.6$ & $17.2 \pm 0.5$ \\
\hline Butanol fraction & $1.5 \pm 0.3$ & $16.3 \pm 0.6$ \\
\hline Water fraction & 0 & $14.1 \pm 0.4$ \\
\hline Control (Tetracycline) & $19.0 \pm 0.4$ & $23.2 \pm 0.5$ \\
\hline
\end{tabular}

\subsection{Morphology of neat and PE extract-loaded CA fiber mat}

Fig 1a) shows a selected SEM image of neat electrospun CA fibers prepared from $17 \% \mathrm{w} / \mathrm{v}$ CA solution in $2: 1 \mathrm{v} / \mathrm{v}$ acetone $/ N, N$-dimethylacetamide under the electrical field of $15 \mathrm{kV} / 15 \mathrm{~cm}$. Cross-sectional round fibers with smooth surface were obtained. The average diameter of these fibers was $335 \pm 52 \mathrm{~nm}$, which is a few larger than that reported by Suwantong et al. [17] (i.e. $301 \pm 64 \mathrm{~nm}$ ).
This could be due to the different size of nozzle used in electrospinning and different electric field.

After dissolving PE extract (diethyl ether fraction) at concentration $1 \%, 3 \%$, and $5 \% \mathrm{w} / \mathrm{w}$ (based on the weight of $\mathrm{CA}$ ) into $17 \% \mathrm{w} / \mathrm{w} \mathrm{CA}$ solutions, the as-prepared solutions were electrospun. The selected SEM images of the PE extract-loaded CA fibers are shown in Fig 1b-d. The surfaces of PE extract-loaded CA fibers were also smooth with no presence of PE extract aggregates. This implies that the PE extracts were incorporated well within the electrospun fibers. It was observed that the average diameters of these fibers were increased with increasing PE extract concentration. The viscosities of the asprepared solutions and the average diameters of electrospun fibers are presented in Table 2. The increasing in the size of fibers upon increasing PE extract concentration could be due to the increasing of viscosity of solution. Viscosity of solution is one of the important factors in controlling size of electrospun fibers. An increasing of viscosity of solution indicated the increasing of viscoelastic force in which a charged jet can withstand more stretching force in electrospinning and therefore resulting in larger fibers.
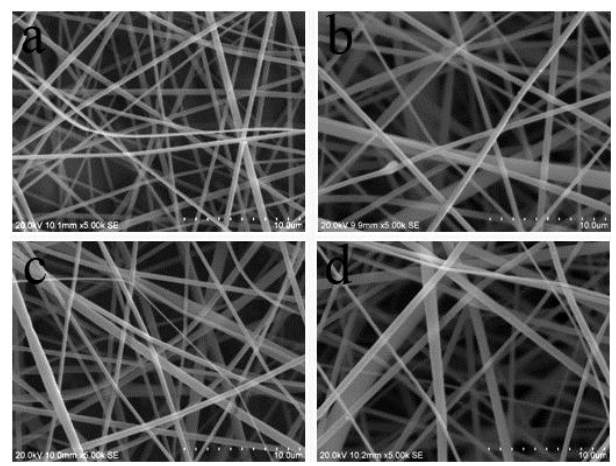

Figure 1. Selected SEM images (x5000) of (a) neat electrospun CA fiber mats. (b) $1 \%$ initial PE extract-loaded electrospun CA fiber mats. (c) 3\% initial PE extract-loaded electrospun CA fiber mats. (d) $5 \%$ initial PE extract-loaded electrospun CA fiber mats.

Table 2. Viscosity of the as-prepared solutions and average diameters of electrospun fibers.

\begin{tabular}{|c|c|c|}
\hline Solutions & $\begin{array}{c}\text { Viscosity } \\
{[\mathrm{cP}]}\end{array}$ & $\begin{array}{c}\text { Average } \\
\text { diameter of } \\
\text { fibers [nm] }\end{array}$ \\
\hline Neat CA solution & $428 \pm 2$ & $335 \pm 52$ \\
1\% PE extract-loaded CA & $442 \pm 3$ & $459 \pm 57$ \\
3\% PE extract-loaded CA & $445 \pm 3$ & $466 \pm 62$ \\
5\% PE extract-loaded CA & $513 \pm 3$ & $694 \pm 87$ \\
\hline
\end{tabular}

\subsection{Water retention of PE extract-loaded CA fiber mats and cast films}

The amounts of water retention of the PE extract-loaded $\mathrm{CA}$ fiber mats and cast films after immersion in acetate buffer (pH 5.5) at $32^{\circ} \mathrm{C}$ for $72 \mathrm{~h}$ is shown in Fig 2. The percentage of water retention of the PE extract-loaded CA fiber mats was in a range of $112-205 \%$, while these values for the cast films was in a range of $25-35 \%$. The 
greater amount of water retention of electrospun fiber mats over that of the cast films should be a result of the highly porous structure of electrospun fibers, therefore much greater surface area when compared with that of the films. These results are consistent with previous studies in which electrospun $\mathrm{CA}$ fiber mats exhibited higher amount of water retention than CA films [16-17]. Comparing among the fiber mat specimens, the amount of water retention of $1 \%$ PE extract-loaded fiber mats was higher than those of $3 \%$ and $5 \%$ PE extract-loaded fiber mats, respectively. This could be a result of difference in the size of fibers. The smaller average fiber diameter of $1 \%$ PE extract-loaded fibers than those of $3 \%$ and 5\% PE extract-loaded fibers could provide more porosity and allow more penetration of media into the specimens. The amount of water retention will be discussed along with the results on release characteristics in the following section.

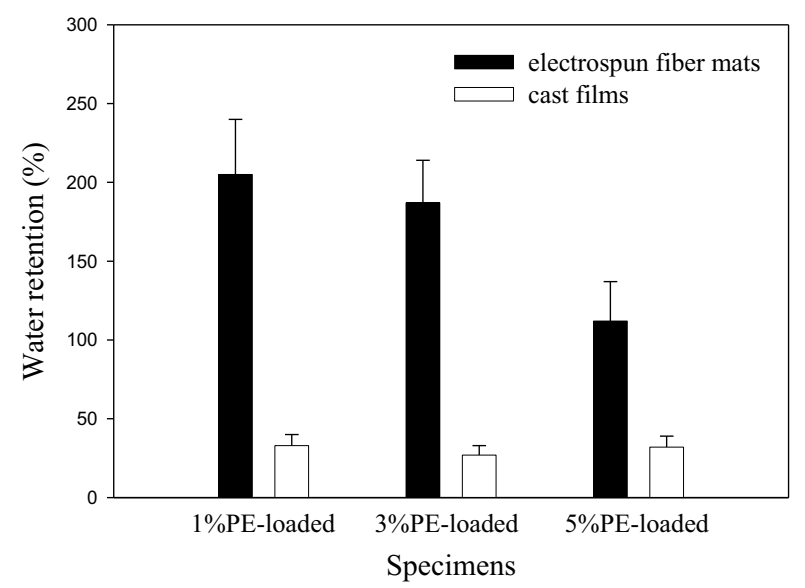

Figure 2. Amount of water retention (\%) of the PE extractloaded CA fiber mats and cast films

\subsection{Release of PE extract-loaded CA fiber mats and cast films}

\subsubsection{Actual PE extracts content}

Prior to investigate the release characteristics of the PE extract from both fiber mats and cast films, the actual amounts of the PE extract within theses samples were determined. These values are illustrated in Table 3 which was reported as the actual amount of PE extract based on the initial amount of PE extract loaded. The actual amounts of PE extract in the PE extract-loaded CA fiber mats were in a range of $87-90 \%$, while these values for the cast films was in a range of $90-92 \%$. These values were slightly deviated from the ideal value of $100 \%$ which could be due to the inhomogeneity of the PE extract in different areas of the samples, even though the samples were carefully prepared.
Table 3. Actual amount of PE extract (\%) in the PE extractloaded CA fiber mats and cast films

\begin{tabular}{|c|c|c|}
\hline \multirow{2}{*}{$\begin{array}{c}\text { Initial amount } \\
\text { of the PE } \\
\text { extract load (\%) }\end{array}$} & \multicolumn{2}{|c|}{ Actual amount of PE extract (\%) } \\
\cline { 2 - 3 } & $\begin{array}{c}\text { PE extract-loaded } \\
\text { CA fiber mats }\end{array}$ & $\begin{array}{c}\text { PE extract-loaded } \\
\text { cast CA films }\end{array}$ \\
\hline 1 & $88.2 \pm 1.5$ & $91.5 \pm 1.7$ \\
3 & $89.7 \pm 2.1$ & $91.1 \pm 2.4$ \\
5 & $87.6 \pm 1.9$ & $90.5 \pm 1.8$ \\
\hline
\end{tabular}

\subsubsection{Release of PE extracts}

The release characteristics of $\mathrm{PE}$ extract from the PE extract-loaded CA fiber mats and cast films were carried out by total immersion method in acetate buffer ( $\mathrm{pH} 5.5)$ at $32^{\circ} \mathrm{C}$ for $72 \mathrm{~h}(4,320 \mathrm{~min})$. Fig 3 . shows the cumulative amounts of PE extract released from the fiber mats and films which reported as the percentage of the weight of $\mathrm{PE}$ extract released over the initial actual weight of PE extract in the sample. Both the PE-extract-loaded CA fiber mats and films exhibited a burst release of PE extract at the initial time $(0-120 \mathrm{~min})$ followed by a sustained release. For the $1 \%, 3 \%$, and $5 \%$ PE extractloaded CA fiber mats, the maximum released amounts of $\mathrm{PE}$ extract were about $79.6 \%, 59.2 \%$, and $40.3 \%$, respectively, while those of the corresponding cast films were about $23.4 \%, 20.9 \%$, and $18.2 \%$, respectively. Evidently, lower values of PE extract released were observed in a case of cast film specimens. A number of factors affect to the rate and the maximum amount of substance release in media, e.g. degree of swelling or amount of water retention, solubility of substance in media, and molecular weight of substance released [18]. The higher amount of PE extract released from the fiber mats compared with the film could be due to the greater amounts of water retention of the fiber mats over those of the films. The highly porous structure of electrospun fibers allowed the easier penetration of media into the specimens which contributed to higher amount of water retention as well as higher amount of substance released.

Comparing among the fiber mat specimens, the amount of PE extract released from 1\% PE extract-loaded fiber mats was higher than those of $3 \%$, and $5 \% \mathrm{PE}$ extract-loaded fiber mats, respectively. This could be resulted from the higher amount of water retention of $1 \%$ PE extract-loaded fiber mats than those of $3 \%$, and $5 \%$ PE extract-loaded fiber mats, respectively. However, comparing among the cast film specimens, the amounts of PE extract released were slightly different because of their similar amounts of water retention. 


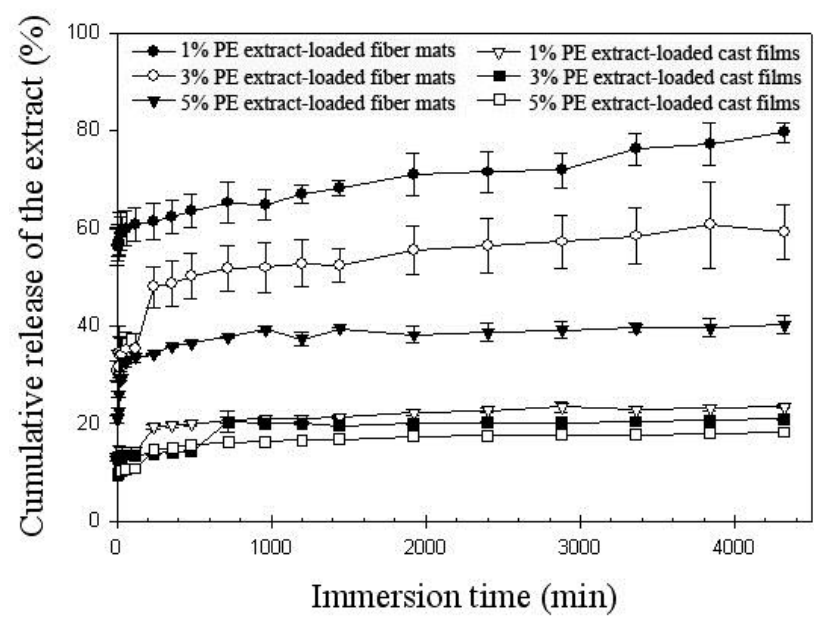

Figure 3. Percentage of cumulative releases of PE extracts from the PE extract-loaded electrospun CA fiber mats and cast films

\subsection{Antibacterial activity of PE extract-loaded CA fiber mats}

The potential of the PE extract-loaded CA fiber mats to be used as transdermal delivery and wound healing is investigated. The antibacterial activity is one of the important properties. The fiber mat specimens were studied for antibacterial activities against $E$. coli by agar disc diffusion method. The mean diameters of inhibition zone of sample are shown in Table 4. It was found that all fiber mat samples showed the diameter of inhibition zone of about $7 \mathrm{~mm}$. This result exhibited that the PE extractloaded CA fiber mats posses the antibacterial property and can be used in the biomedical applications such as transdermal drug delivery and treatment of wound healing.

Table 4. Antibacterial activities of the PE extract-loaded electrospun CA fiber mats against $E$. coli

\begin{tabular}{|c|c|}
\hline Sample & $\begin{array}{c}\text { Mean diameter of } \\
\text { inhibition zone }[\mathrm{mm}]\end{array}$ \\
\hline 1\% PE extract-loaded & $7.1 \pm 0.1$ \\
\hline 3\% PE extract-loaded & $7.1 \pm 0.1$ \\
\hline 5\% PE extract-loaded & $7.0 \pm 0.1$ \\
\hline Control (Tetracycline) & $21.3 \pm 0.3$ \\
\hline
\end{tabular}

\section{Conclusions}

In this work, PE fruit was extracted with methanol and partitioned into diethyl ether, ethyl acetate, butanol, and water fractions. The diethyl ether fraction showed the highest antibacterial activity against $E$. coli and $S$. aureus. Only diethyl ether fraction was then added into CA solutions to prepare the PE extract-loaded electrospun CA nanofibers. The sizes of nanofibers were larger when the concentrations of PE extract increased. The average diameters of nanofibers were in a range of 335-694 nm. The amount of water retention of the PE extract-loaded fiber mats was higher than that of the cast films due to highly porous structure of the electrospun fibers in comparison with the dense structure of films. The release of PE extract was carried out by a total immersion method in an acetate buffer $(\mathrm{pH} 5.5)$ at $32^{\circ} \mathrm{C}$ for $72 \mathrm{~h}$. The cumulative amount of PE extract released from both fiber mats and films increased rapidly during the initial time, and then it increased slowly as the immersion time increased further. The maximum amounts of PE extract released from fiber mats and films were about $40-80 \%$ and $18-23 \%$, respectively. The obtained PE extractloaded fiber mats exhibited antibacterial activities which imply the great potential in biomedical applications such as drug delivery and wound healing.

\section{References}

1. W. Luo, M. Zhao, B. Yang, J. Ren, G. Shen, G. Rao: Food Chem. 126, 277-282 (2010)

2. X. Liu, M. Zhao, W. Luo, B. Yang, Y. Jiang: J Med Food. 12(2), 423-428 (2009)

3. R. Dabur, A. Gupta, T.K. Mandal, D.D. Singh, V. Bajpai, A.M. Gurav, G.S. Lavekar: Afr J Trad Complement Alt Med. 4(3), 313-318 (2007)

4. A. Bhattacharya, A. Chatterjee, S. Ghosal, S.K. Bhattacharya: Indian J Exp Biol. 37, 676-680 (1999)

5. P. Mayachiew, S. Devahastin: LWT-Food Sci Technol. 41, 1153-1159 (2008)

6. M.H. Mehmood, H.S. Siddiqi, A.H. Gilani: J Ethnopharmacol. 133, 856-865 (2011)

7. D.A. Dhale, U.P. Mogle: Sci Res Reporter. 1(3), 138-142 (2011)

8. A. Ghosh, B.K. Das, A. Roy, B. Mandal, G. Chandra: J Nat Med. 62, 259-262 (2008)

9. A. Ihantola-Vormisto, J. Summanen, H. Kankanranta, H. Vuorela, Z.M. Asmawi, E. Moilanen : Planta Med. 63(6), 518-524 (1997)

10. P.W. Gibson, H.L. Schreuder-Gibson, D. Rivin: AIChE J. 45(1), 190-195 (1999)

11. J. Kim, D.H. Reneker: Polym Composite. 20(1), 124131 (1999)

12. P. Wutticharoenmongkol, P. Pavasant, P. Supaphol: Biomacromol. 8(8), 2602-2610 (2007)

13. P. Taepaiboon, U. Rungsardthong, P. Supaphol: Eur J Pharm Biopharm. 67, 387-397 (2007)

14. S. Tungprapa, I. Jangchud, P. Supaphol: Polymer. 48, 5030-5041 (2007)

15. H. Liu, Y.-L. Hsieh: J Polym Sci Pol Phys. 40, 21192129 (2002)

16. P. Thitiwongsawet, P. Ouykul, A. Khaoroppan: Asean J Chem Eng. 10(2), 40-47 (2010)

17. O. Suwantong, P. Opanasopit, U. Ruktanonchai, P. Supaphol: Polymer, 48, 7546-7557 (2007)

18. R. Rujiravanit, S. Kruaykitanon, A.M. Jamieson, S. Tokura: Macromol Biosci. 3, 604-611 (2003) 